\title{
Expression and functional analysis of TCF4 isoforms in human glioma cells
}

 \\ ${ }^{1}$ Department of Clinical Medicine, Graduate School of Southern Medical University, Guangzhou, Guangdong 510515; \\ ${ }^{2}$ Department of Neurosurgery, Wuhan General Hospital of PLA, Wuhan, Hubei 430070; ${ }^{3}$ Department of Neurosurgery, \\ The First Affiliated Hospital of Chongqing Medical University, Chongqing 400016; ${ }^{4}$ Hubei Key Laboratory of Central \\ Nervous System Tumor and Intervention, Wuhan General Hospital of PLA, Wuhan, Hubei 430070, P.R. China
}

Received November 9, 2016; Accepted October 2, 2017

DOI: $10.3892 / \mathrm{mmr} .2018 .8553$

\begin{abstract}
Transcription factor 4 (TCF4) is a member of the T-cell factor/lymphoid enhancer factor (TCF/LEF) transcription factor family in the Wnt/ $\beta$-catenin signaling pathway. The alternative splicing of TCF4 has been reported to exhibit potential carcinogenic properties in various cancer types. In the present study, TCF4 isoforms were cloned and identified in three human glioma cell lines, with the majority of splicing regions being exons 4, 5, 14, 15, and 16. Using MTT assays, it was demonstrated that the overexpression of TCF4 isoforms inhibits the proliferation of U251 cells. Flow cytometry and wound healing analyses revealed that the overexpression of TCF4 isoforms induced cell apoptosis and migration. Taken together, the $\beta$-catenin binding domain of the TCF4 isoforms inhibited cell proliferation, and induced cell apoptosis and migration in glioma. Furthermore, all the isoforms identified contained the $\mathrm{N}$-terminal part of TCF4 including the $\beta$-catenin binding domain. This implied that a high expression of TCF4 isoforms may lead to Wnt/ $\beta$-catenin signal activation and potentially promote malignant glioma development.
\end{abstract}

\section{Introduction}

Gliomas are the most common intrinsic brain tumors, which make up $\sim 30 \%$ of all brain and central nervous system tumors and $80 \%$ of all malignant brain tumors (1). They are characterized by high morbidity, high recurrence rates, high mortality and low cure rate (2). In recent years with the development

Correspondence to: Dr Guo-Zheng Xu, Department of Clinical Medicine, Graduate School of Southern Medical University, 1063 Shatai Road, Guangzhou, Guangdong 510515, P.R. China E-mail: gzxu16@sina.com

Mr Bo Diao, Hubei Key Laboratory of Central Nervous System Tumor and Intervention, Wuhan General Hospital of PLA, 627 Wuluo Road, Wuchang, Wuhan, Hubei 430070, P.R. China E-mail: dpitao@163.com

Key words: TCF4, isoform, Wnt//-catenin, glioma of imaging diagnosis and microneurosurgery, diagnosis and treatment of gliomas has made some progress but little breakthrough in the treatment of malignant gliomas, most of patients die within 1 year of diagnosis $(3,4)$. Despite the frequency of gliomas, the etiology that contributes to tumor initiation remains to be elucidated. Previous studies revealed that aberrant regulation of the Wnt signaling pathway has a vital role in the process of glioma and malignant progression (5-7).

Wnt/ $\beta$-catenin signaling pathway has been reported to be involved in the regulation of life processes, including cell proliferation, cell apoptosis, organ growth and development, cancer and inflammatory diseases $(8,9)$. The canonical Wnt pathway consists of Wnt protein, frizzled family receptor, dishevelled, glycogen synthase kinase $3 \beta$, adenomatous polyposis coli, axin, $\beta$-catenin and $\mathrm{T}$ cell transcriptional regulation factors (TCF). It is activated by binding a Wnt-protein ligand to a frizzled family receptor, which passes the biological signal to the downstream pathway inside the cells. Following activation by external stimulation, $\beta$-catenin is translocated into nucleus to combine with TCF, followed by initiation of transcription regulation of target genes such as Myc proto-oncogene, bHLH transcription factor, cyclin D1 and cytochrome $c$ oxidase subunit II, which are involved in cell apoptosis and may have carcinogenic effects $(9,10)$.

TCF4 and $\beta$-catenin are upregulated in gliomas compared with the normal brain, inhibiting cell proliferation and inducing cell apoptosis (11). TCF4, one of TCFs factors, including lymphoid enhancer-binding factor (LEF1), TCF1, TCF3 and TCF4, has been previously reported to exhibit high expression in renal carcinoma, colorectal cancer, hepatocellular carcinoma and brain tumors (11-16). Abnormal upregulation of TCF4 stimulating downstream target genes is a common early event in tumorigenesis.

The human TCF4 gene consists of 17 exons with several alternatively splicing sites, including a C-terminal tail (exon 13-17) and exon 4 (16). Previous studies revealed that TCF mRNAs were subject to alternative splicing to form different isoforms, which are also important in regulating transcription in the Wnt signaling pathway and associated with tumorigenesis. Different TCF4 isoforms have been identified in various types of cancer including renal carcinoma (14), colorectal cancer (16) and brain tumor (17). However, the importance 
Table I. Sequences of primers used in the present study.

\begin{tabular}{lcll}
\hline Primer & Exon & Direction & \multicolumn{1}{c}{ Sequence (5'-3') } \\
\hline H251 TCF4 & 10 & Forward & AGTGCACGTTGAAAGAAAGCGCG \\
H252 TCF4 & 17 SR & Reverse & CTGCCTTCACCTTGTATGTAGAG \\
H557 TCF4 & 1 & Forward & CTTCCAAAATTGCTGCTGGTG \\
H558 TCF4 & 11 & Reverse & TCTCTGGACAGTGCATGCC \\
H559 TCF4 & 12 & Forward & CAAGCAGCCGGGAGAGAC \\
H562 TCF4 & 15 & Forward & ATGCAAATACTCCAAAGAAG \\
H563 TCF4 & $17 \mathrm{LR}$ & Reverse & TCAGCGAGCAGGAGGC \\
H580 TCF4 & 15 & Reverse & CTGCACGGTTTGCACCAT \\
\hline
\end{tabular}

TCF4, transcription factor 4 .

of TCF4 isoforms in the process of gliomas remains to be elucidated. The present study analyzed the alterative splicing forms of TCF4 and characterized their biological functions in three different glioma cell lines. The findings of the present study provide evidence that TCF4 isoforms may contribute to human gliomas, which lay the foundation for molecular mechanisms of regulation of malignant biological behavior of glioma.

\section{Materials and methods}

Cell culture. U251, A172 and U-87MG human glioma cell lines (Type Culture Collection of the Chinese Academy of Sciences, Shanghai, China) were maintained in Dulbecco's modified Eagle's medium (DMEM) supplemented with 10\% fetal bovine serum (FBS) (both from Gibco; Thermo Fisher Scientific, Inc., Waltham, MA, USA), $100 \mathrm{U} / \mathrm{ml}$ penicillin and $100 \mu \mathrm{g} / \mathrm{ml}$ streptomycin and were maintained at $37^{\circ} \mathrm{C}$ and a $5 \% \mathrm{CO}_{2}$ humidified environment.

RNA extraction, reverse transcription-polymerase chain reaction $(R T-P C R)$ and sequence alignment. Total RNA was isolated from cells using TRIzol reagent (Invitrogen; Thermo Fisher Scientific, Inc.), and cDNA was synthesized using PrimeScript II First-Strand cDNA Synthesis kit (Takara Bio, Inc., Otsu, Japan) at $42^{\circ} \mathrm{C}$ for $60 \mathrm{~min}$ and $95^{\circ} \mathrm{C}$ for $5 \mathrm{~min}$. Subsequently, cDNA sequences were amplified by Premix Taq (Takara Bio, Inc.) with specific primers presented in Table I at $98^{\circ} \mathrm{C}$ for $10 \mathrm{sec}, 58^{\circ} \mathrm{C}$ for $30 \mathrm{sec}, 72^{\circ} \mathrm{C}$ for $3 \mathrm{~min}$ and $72^{\circ} \mathrm{C}$ for $5 \mathrm{~min}$. DNA fragments were isolated from the agarose gels with Minibest Agarose Gel Extraction kit (Takara Bio, Inc.). PCR products were then subcloned into T-vector PMD19-T (Takara Bio, Inc.) and an individual clone was selected for sequencing by Sangon Biotech Co., Ltd., (Shanghai, China). Sequence alignments were performed with TCF4 genome (gene ID: 6934) deposited in Genbank by BLAST (blast.ncbi. nlm.nhi.gov/Blast.cgi).

Construction of expression vectors and cell transfection. Three TCF4 isoforms (TCF4VX35, TCF4VX18 and TCF4V7) were inserted into the corresponding site of the pVITRO2-neo-mcs vector (Takara Bio, Inc.) site. Cells $\left(2 \times 10^{5}\right.$ cells $\left./ \mathrm{ml}\right)$ were seeded in 24-well plates and cultured until the cells were at $70-80 \%$ confluence, subsequently the cells were transfected with $800 \mathrm{ng} /$ well of expression plasmids using Lipofectamine 2000 (Invitrogen; Thermo Fisher Scientific, Inc.) as specified by the manufacturer's protocol. After $48 \mathrm{~h}$, green fluorescence was examined under a fluorescence microscope (Olympus Corporation, Tokyo, Japan).

MTT assay. Cell proliferation was determined using an MTT assay. U251 cells $\left(4 \times 10^{4}\right.$ cells $\left./ \mathrm{ml}\right)$ were seeded in 96-well plates and transfected with expression vectors or empty vector (Takara Bio, Inc.). At $48 \mathrm{~h}$ following transfection, $20 \mu \mathrm{l}$ MTT ( 5 mg/ml; Sigma-Aldrich; Merck KGaA, Darmstadt, Germany) was added to each culture and incubated for 4-6 h. At the end of the incubation period the medium were removed and $150 \mu \mathrm{l}$ DMSO was added to each well. After agitation at a low speed for $10 \mathrm{~min}$, absorbance of converted dye was measured at a wavelength of $490 \mathrm{~nm}$.

Flow cytometry. Apoptosis analysis was performed with the Annexin V-PE/7-AAD flow cytometry kit (BD Biosciences, Franklin Lakes, NJ, USA) according to the manufacturer's protocol. U251 cells $\left(1.5 \times 10^{6}\right.$ cells $\left./ \mathrm{ml}\right)$ in 6 -well plate were transfected with expression vectors or empty vector of TCF4 isoforms using Lipofectamine 2000. After 48 h, cells were washed twice with ice-cold PBS and resuspended in $1 \mathrm{X}$ binding buffer at a concentration of $1 \times 10^{6}$ cells $/ \mathrm{ml}$. The solution $(100 \mu \mathrm{l})$ was transferred to a $5 \mathrm{ml}$ culture tube. PE Annexin V $(5 \mu \mathrm{l})$ and $5 \mu \mathrm{l}$ 7-AAD was added and cells were incubated for $15 \mathrm{~min}$ at $25^{\circ} \mathrm{C}$ in the dark. Finally, $400 \mu \mathrm{l} 1 \mathrm{X}$ binding buffer was added to each tube and was analyzed by FC500MCL flow cytometry using CXP (version 2.2; Beckman Coulter, Inc., Brea, CA, USA) within $1 \mathrm{~h}$.

Wound healing assay. Cell migration was assessed using a scratch wound assay. U251 cells $\left(1 \times 10^{6}\right.$ cells $\left./ \mathrm{ml}\right)$ were grown to $60-70 \%$ confluence and transfected with expression vectors of TCF4 isoforms using Lipofectamine 2000. After $24 \mathrm{~h}$, a linear wound was made by scraping a sterile micropipette tip across the confluent cell layer. Cells were washed three times with PBS to remove detached cells and debris. Subsequently the size of wounds was observed and measured on a TS100-F inverted-phase contrast microscope (Nikon Corporation, Tokyo, Japan) at 0 and $48 \mathrm{~h}$. 


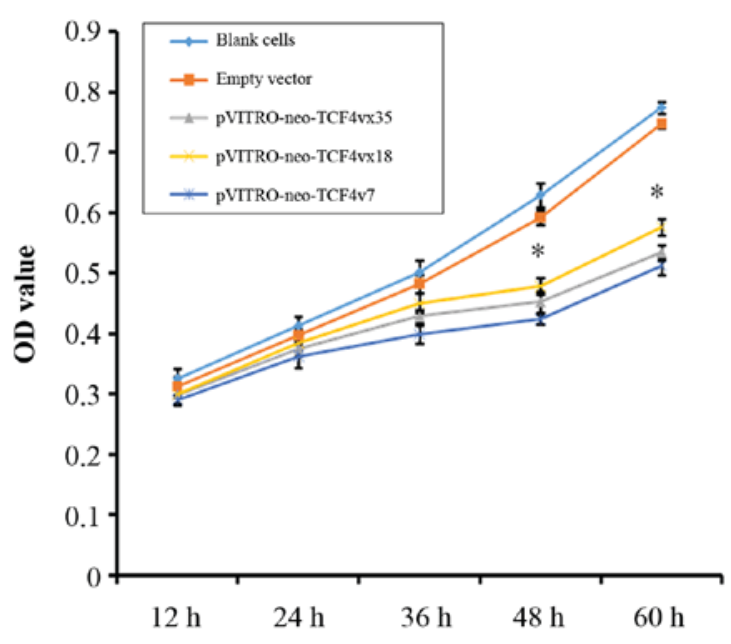

Figure 1. Overexpression of TCF4 isoforms inhibited cell proliferation. Each TCF4 isoform expression plasmid was transiently transfected into U251 cells and an OD value was determined for $60 \mathrm{~h}$. Blank cells and an empty vector were used as controls. Cells transfected with TCF4 isoforms had a reduced growth rate. ${ }^{*} \mathrm{P}<0.001$ vs. control $(\mathrm{n}=3)$. OD, optical density; TCF4, transcription factor 4 .

Statistical analysis. Data are expressed as the mean \pm standard error of the mean. Statistical analysis was performed using a one-way analysis of variance followed by Duncan's multiple range test as a post-hoc test. $\mathrm{P}<0.05$ was considered to indicate a statistically significant difference.

\section{Results}

Determination of TCF4 isoforms in human glioma cell lines. TCF4 isoforms have been identified in various tumor cells in the majority of body systems and organs $(14,15,18)$. In order to determine whether there are TCF4 isoforms in human glioma cells, nest PCR analysis using specific primers of human TCF4 in three cell lines (U251, A172 and U-87MG) was performed. PCR products were inserted into a PMD19-T vector and an individual clone was selected to be sequenced. A total of 13 different TCF isoforms were expressed in U251 cells in the present study, including 3 isoforms (vx35, vx18 and v7), which had high scores after blasting the TCF genome in the NCBI database. A total of 19 different TCF isoforms were expressed in A172 cells and 3 isoforms (18, 27 and vx21) gained top scores. In the U-87MG cells 12 different TCF isoforms were expressed and 3 isoforms (27, 18 and vx21) had the highest scores. They had several distinct features: v7 was found to lack exon 4, 6, whereas vx18 lacked exon 4, 6, 14, 15, vx21 lacked part of exon 17 and had an additional amino acid, vx27 lacked exon 14 and part of exon 17 and vx35 lacked exon 4, 6, 15 and 16. The present study selected vx35, vx18 and v7 found in U251 cells for further study. The three TCF4 isoforms (TCF4VX35, TCF4VX18 and TCF4V7) were inserted into the pVITRO2-neo-mcs vectors. The eukaryotic expression plasmids pVITRO-neo-TCF4vx35, pVITRO-neo-TCF4vx18 and pVITRO-neo-TCF4vx7 were successfully expressed in U215 cells.

Effect of TCF4 isoforms on cell proliferation. TCF4 may serve different roles in different tumor cells. Overexpression
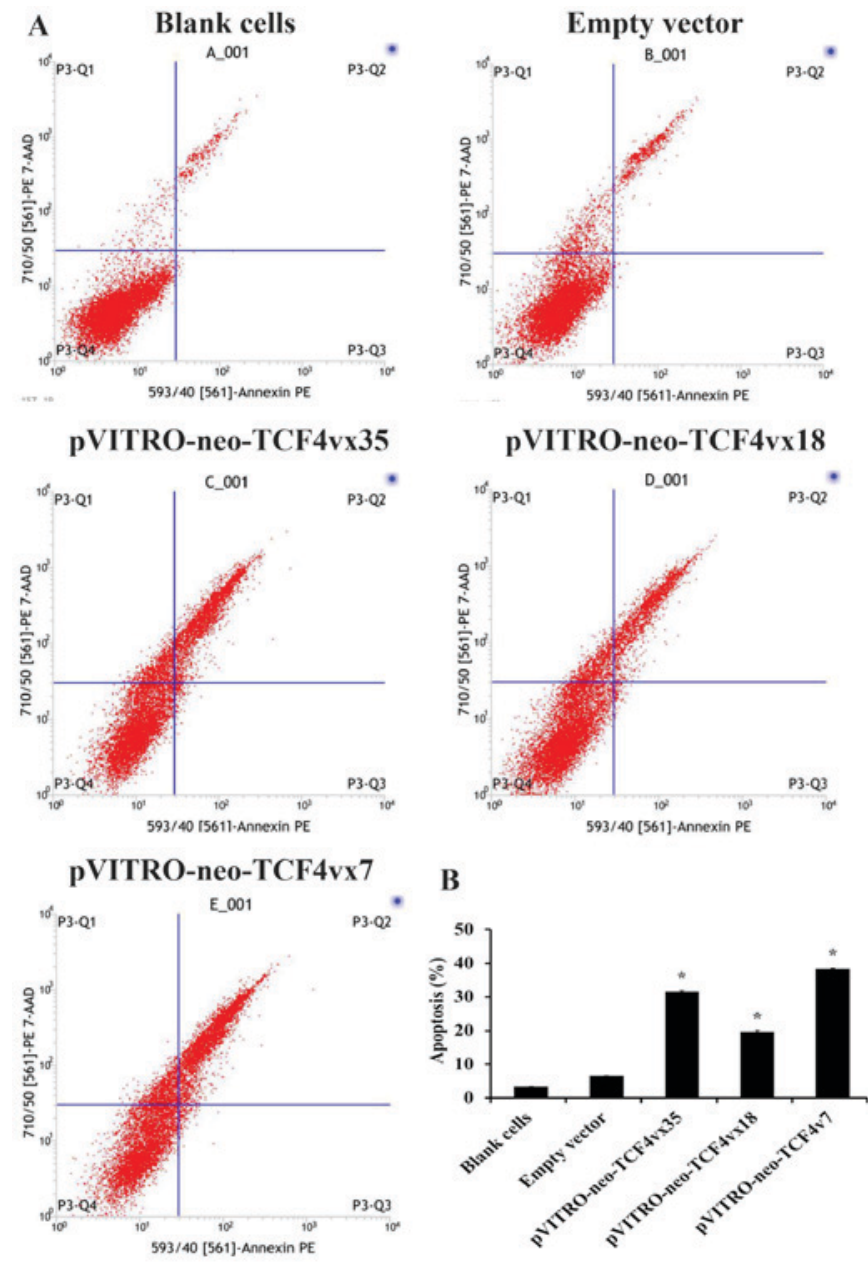

Figure 2. Overexpression of TCF4 isoforms induced cell apoptosis. (A) Cell apoptosis was measured by staining with Annexin V/7-AAD using flow cytometry. Cells were incubated with PE Annexin V in a buffer containing 7-AAD and analyzed by flow cytometry. Untreated cells were primarily PE Annexin V and 7-AAD negative, indicating that they were viable and not undergoing apoptosis. (B) Apoptosis of cells transfected with pVITRO-neo-TCF4vx35, pVITRO-neo-TCF4vx18 or pVITRO-neo-TCF4vx7 compared to empty vector control was significant. ${ }^{*} \mathrm{P}<0.001$ vs. empty vector control. 7-AAD, 7-amino-actinomycin D; P3-Q1, dead cells; P3-Q2, late stage apoptosis; P3-Q3, early stage apoptosis; P3-Q4, viable cells; TCF4, transcription factor 4 .

of the TCF4 isoforms has been previously associated with oncogenesis and development of malignant tumors $(11,18,19)$. In order to investigate the effect of TCF4 isoforms on U251 cell proliferation, each TCF4 isoform expression plasmid was transiently transfected into cells and growth rate was measured from 12 to $60 \mathrm{~h}$. As presented in Fig. 1, pVITRO-neo-TCF4vx35, pVITRO-neo-TCF4vx18 and pVITRO-neo-TCF4vx7 groups had reduced cell proliferation compared with the empty vector control at 48 and $60 \mathrm{~h}$ $(\mathrm{P}<0.001)$.

Effect of TCF4 isoforms on cell apoptosis. Following the observation that TCF4 isoforms inhibited cell growth rate, the present study aimed to determine whether TCF4 isoforms induced cell apoptosis. The Annexin V-PE/7-AAD flow cytometry was performed for apoptosis analysis. As presented in Fig. 2, U251 cells transfected with pVITRO-neo-TCF4vx35, pVITRO-neo-TCF4vx18 and pVITRO-neo-TCF4vx7 had 


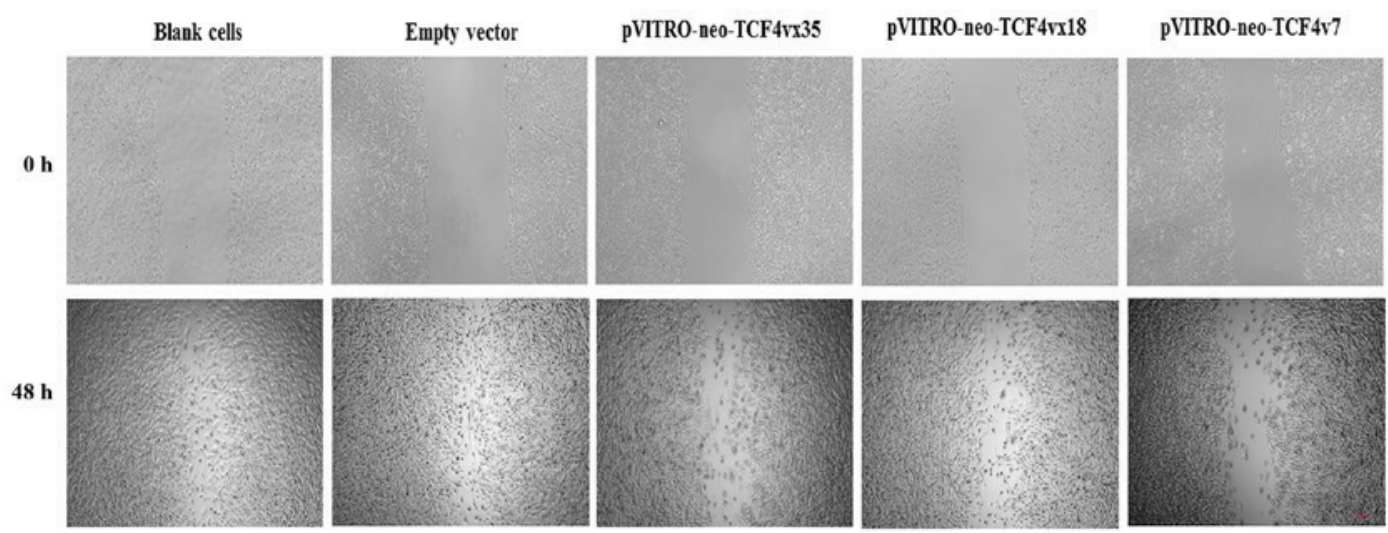

Figure 3. Overexpression of TCF4 isoforms induced cell migration. A wound healing assay was performed to analyze migration. Cells transfected with pVITRO-neo-TCF4vx35, pVITRO-neo-TCF4vx 18 or pVITRO-neo-TCF4vx7 were compared with the empty vector at $48 \mathrm{~h}$ after transfection. TCF4, transcription factor 4 .

$31.59,19.60$ and $38.43 \%$ more apoptotic cells compared with the empty vector control (6.67\%; P<0.001; Fig. 2B).

Effect of TCF4 isoforms on cell migration. To determine if the TCF isoforms have a function in cell migration, a wound healing assay was performed. As presented in Fig. 3, U251 cells overexpressing pVITRO-neo-TCF4vx35, pVITRO-neo-TCF4vx18 and pVITRO-neo-TCF4vx7 had increased cell migration; however, this was decreased compared with the control.

\section{Discussion}

The Wnt signaling pathway is involved in the development and homeostasis of cells and tissues, and aberrant regulation Wnt signal frequently occurs during the initiation of various tumor types (8). TCF proteins may act as transcriptional activators or repressors in nucleus in the downstream in Wnt signaling. It has been previously reported that TCF4 and its splice variants are critical determinants of context-dependent Wnt signaling responses, including physiological development or oncogenesis $(20,21)$. Previous studies revealed that human TCF4 gene was alternatively spliced in various types of cancers, including brain tumors (17). The present study aimed to determine the TCF4 isoforms that may be associated with biological properties of glioma cells and the development of the malignant phenotype.

Differential expression and splicing isoform analysis of TCF4 has been previously reported in the tissue of brain tumors (17). However, to the best of our knowledge, there is no report on gliomas regarding the TCF4 isoform and its significance in tumorigenesis. The present study analyzed TCF4 isoforms in three human glioma cell lines. In the TCF4 gene, three major domains are present: $\beta$-catenin-binding domain in exon 1, the DNA-binding HMG boxes in exons 10 and 11, and the $\mathrm{COOH}$-terminal-binding domain in exon $17(22,23)$. The current study identified 13 different TCF4 isoforms in U251 cells, 19 different TCF isoforms in A172 cells and 12 different TCF isoforms in U-87MG cells. It was previously demonstrated that the majority of alternative splicing occurred within exon 12-17 in brain tumor (17). The present study revealed that the majority of alternative spliced regions were exon 4, 5, 14,
15 and 16 in glioma cells. However, in colorectal cancer cell lines various splicing isoforms of TCF4 mRNA were present in the $\mathrm{COOH}$-terminal region (exon 17) (16). In renal cell carcinomas, four splicing isoforms of the TCF4 were present in the region between exon 12 and exon 17 (14). All the isoforms the current study identified contained the $\mathrm{N}$-terminal part of TCF4 including the $\beta$-catenin binding domain; therefore, they may not lack the transcriptional activity and Wnt/ $\beta$-catenin was activated in glioma cells to contribute to tumorigenesis. However, the specific molecular mechanism remains to be elucidated.

To evaluate the functional properties of TCF4 isoforms in U251 cells, TCF4 isoform expression plasmid was transfected and cell proliferation, apoptosis and migration were quantified. The findings of the current study revealed that the isoforms may inhibit the proliferation and induce the apoptosis and migration of U251 cells. After Wnt signaling transduction cascade is triggered, cytoplasmic $\beta$-catenin translocates to nucleus to bind TCF and modulates expression of target genes critical for cell proliferation, differentiation, survival and apoptosis (24). It is possible that overexpression of TCF isoforms may lead to activation of $\beta$-catenin/TCF transcription for target genes associated with cell proliferation and apoptosis. Conversely, cells lose cell polarity and cell-cell adhesion, and gain migratory and invasive properties during the epithelial-mesenchymal transition, which is induced by $\mathrm{Wnt} / \beta$-catenin $(25,26)$. Overexpression of TCF isoforms appears to be sufficient to induce cell migration via the Wnt/ $\beta$-catenin pathway. However, the function and malignant biological behaviors of TCF4 isoforms require further investigation.

In conclusion, the TCF4 isoforms in three human glioma cells lines were cloned and identified in the present study. The functions and properties of TCF4 isoforms were analyzed in U251 cells. The findings of the present study revealed that TCF4 isoforms inhibited cell proliferation and induced the cell apoptosis and migration. Therefore, this mechanism of Wnt/ $\beta$-catenin-mediated TCF isoforms transcription may regulate the target genes that contribute to the tumorigenesis of glioma. Due to the carcinogenic properties of the TCF isoforms, the interaction between $\mathrm{Wnt} / \beta$-catenin and the TCF isoforms should be assessed in future studies. 


\section{Acknowledgements}

The current study was supported by grants from Hubei Province Health and Family Planning Scientific Research Project (grant no. WJ2015MB118) and Medical Scientific Research Foundation of Hubei Province, China (grant no. 2016CFC745).

\section{References}

1. Goodenberger ML and Jenkins RB: Genetics of adult glioma. Cancer Genet 205: 613-621, 2012.

2. Reuss D and von Deimling A: Hereditary tumor syndromes and gliomas. Recent Results Cancer Res 171: 83-102, 2009.

3. Riezzo I, Zamparese R, Neri M, De Stefano F, Parente R, Pomara C, Turillazzi E, Ventura F and Fineschi V: Sudden, unexpected death due to glioblastoma: Report of three fatal cases and review of the literature. Diagn Pathol 8: 73, 2013.

4. Swanson KD, Lok E and Wong ET: An overview of alternating electric fields therapy (NovoTTF Therapy) for the treatment of malignant glioma. Curr Neurol Neurosci Rep 16: 8, 2016.

5. Sareddy GR, Panigrahi M, Challa S, Mahadevan A and Babu PP: Activation of $\mathrm{Wnt} /$ beta-catenin/Tcf signaling pathway in human astrocytomas. Neurochem Int 55: 307-317, 2009.

6. Schüle R, Dictus C, Campos B, Wan F, Felsberg J, Ahmadi R, Centner FS, Grabe N, Reifenberger G, Bermejo JL, et al: Potential canonical wnt pathway activation in high-grade astrocytomas. ScientificWorldJournal 2012: 697313, 2012.

7. Zhang J, Huang K, Shi Z, Zou J, Wang Y, Jia Z, Zhang A, Han L, Yue X, Liu N, et al: High $\beta$-catenin/Tcf-4 activity confers glioma progression via direct regulation of AKT2 gene expression. Neuro Oncol 13: 600-609, 2011

8. Duchartre Y, Kim YM and Kahn M: The Wnt signaling pathway in cancer. Crit Rev Oncol Hematol 99: 141-149, 2016.

9. Clevers $\mathrm{H}$ and Nusse R: Wnt $/ \beta$-catenin signaling and disease. Cell 149: 1192-1205, 2012

10. Kikuchi A: Canonical Wnt signaling pathway and cellular responses. Clin Calcium 23: 799-807, 2013 (In Japanese).

11. Chen L, Huang K, Han L, Shi Z, Zhang K, Pu P, Jiang C and Kang C: $\beta$-catenin/Tcf- 4 complex transcriptionally regulates AKT1 in glioma. Int J Oncol 39: 883-890, 2011.

12. Travis A, Amsterdam A, Belanger C and Grosschedl R: LEF-1, a gene encoding a lymphoid-specific protein with an HMG domain, regulates T-cell receptor alpha enhancer function [corrected]. Genes Dev 5: 880-894, 1991.

13. van de Wetering M, Oosterwegel M, Dooijes D and Clevers H: Identification and cloning of TCF-1, a T lymphocyte-specific transcription factor containing a sequence-specific HMG box. EMBO J 10: 123-132, 1991.
14. Shiina H, Igawa M, Breault J, Ribeiro-Filho L, Pookot D, Urakami S, Terashima M, Deguchi M, Yamanaka M, Shirai M, et al: The human T-cell factor-4 gene splicing isoforms, Wnt signal pathway, and apoptosis in renal cell carcinoma. Clin Cancer Res 9: 2121-2132, 2003.

15. Tsedensodnom O, Koga H, Rosenberg SA, Nambotin SB Carroll JJ, Wands JR and Kim M: Identification of T-cell factor-4 isoforms that contribute to the malignant phenotype of hepatocellular carcinoma cells. Exp Cell Res 317: 920-931, 2011.

16. Duval A, Rolland S, Tubacher E, Bui H, Thomas G and Hamelin R: The human T-cell transcription factor-4 gene: Structure, extensive characterization of alternative splicings, and mutational analysis in colorectal cancer cell lines. Cancer Res 60: 3872-3879, 2000.

17. Howng SL, Huang FH, Hwang SL, Lieu AS, Sy WD, Wang C and Hong YR: Differential expression and splicing isoform analysis of human Tcf-4 transcription factor in brain tumors. Int J Oncol 25: 1685-1692, 2004.

18. He G, Guan X, Chen X, Wang Y, Luo C and Zhang B: Expression and splice variant analysis of human TCF4 transcription factor in esophageal cancer. J Cancer 6: 333-341, 2015.

19. Gu X, Yao L, Ma G, Cui L, Li Y, Liang W, Zhao B and Li K: TCTP promotes glioma cell proliferation in vitro and in vivo via enhanced $\beta$-catenin/TCF-4 transcription. Neuro Oncol 16: 217-227, 2014.

20. Wallmen B, Schrempp M and Hecht A: Intrinsic properties of Tcf 1 and Tcf 4 splice variants determine cell-type-specific Wnt/ $\beta$-catenin target gene expression. Nucleic Acids Res 40: 9455-9469, 2012

21. Tomimaru Y, Koga H, Yano H, de la Monte S, Wands JR and Kim M: Upregulation of T-cell factor-4 isoform-responsive target genes in hepatocellular carcinoma. Liver Int 33: 1100-1112, 2013.

22. Clevers $\mathrm{H}$ and van de Wetering $\mathrm{M}$ : TCF/LEF factor earn their wings. Trends Genet 13: 485-489, 1997.

23. Duval A, Gayet J, Zhou XP, Iacopetta B, Thomas G and Hamelin R: Frequent frameshift mutations of the TCF-4 gene in colorectal cancers with microsatellite instability. Cancer Res 59: 4213-4215, 1999

24. Barker N: The canonical Wnt/beta-catenin signalling pathway. Methods Mol Biol 468: 5-15, 2008.

25. Micalizzi DS and Ford HL: Epithelial-mesenchymal transition in development and cancer. Future Oncol 5: 1129-1143, 2009.

26. Gonzalez DM and Medici D: Signaling mechanisms of the epithelial-mesenchymal transition. Sci Signal 7: re8, 2014. 